\title{
Adiabatic Evolution of Coupled Waves for a Schrödinger-Korteweg-de Vries System
}

\author{
W. Abou Salem* \\ Department of Mathematics and Statistics, University of Saskatchewan, SK S7N 5E6, Canada
}

\begin{abstract}
The effective dynamics of interacting waves for coupled Schrödinger-Korteweg-de Vries equations over a slowly varying random bottom is rigorously studied. One motivation for studying such a system is better understanding the unidirectional motion of interacting surface and internal waves for a fluid system that is formed of two immiscible layers. It was shown recently by Craig-Guyenne-Sulem [1] that in the regime where the internal wave has a large amplitude and a long wavelength, the dynamics of the surface of the fluid is described by the Schrödinger equation, while that of the internal wave is described by the Korteweg-de Vries equation. The purpose of this letter is to show that in the presence of a slowly varying random bottom, the coupled waves evolve adiabatically over a long time scale. The analysis covers the cases when the surface wave is a stable bound state or a long-lived metastable state.
\end{abstract}

Keywords and phrases: Korteweg-de Vries equation, Schrödinger equation, coupled waves, effective dynamics, adiabatic theorem

Mathematics Subject Classification: 35Q35, 35Q40, 35Q51, 81Q99

\section{Introduction}

\subsection{Motivation and heuristic discussion}

We study the adiabatic evolution of interacting waves for coupled Schrödinger-Korteweg-de Vries equations over a slowly varying random bottom. The main physical motivation for the analysis is understanding the effective dynamics of coupled surface and internal waves in a fluid system where two immiscible layers are separated by a sharp free interface, such as thermocline or pycnocline differential salinity in the ocean. Recent photographs from the space shuttle showed bright trains or strips of waves over several kilometers in the Andaman sea, which is a signature of interaction between surface and internal waves. The heuristic derivation of effective equations that describe this observation was done by Craig-GuyenneSulem [1]. In the regime where the wavelength of the internal wave is long and its amplitude is large, it was shown in the latter that the unidirectional dynamics of the surface wave is described by the linear Schrödinger equation, while that of the internal wave is described by the KdV equation. The formal analysis in [1] starts from the Hamiltonian formulation of Euler equations for an irrotational fluid and uses perturbation theory for the modulated regime, as in [2]. Here, we modify the KdV equation describing the

\footnotetext{
${ }^{*}$ Corresponding author. E-mail: abousalem@math.usask.ca
} 
dynamics of the internal wave by adding an effective term to include the effect of a slowly varying bottom. Such a term appears in the derivation of the KdV equation over a slowly varying bottom, [3, 4], and has been used to study the long-time dynamics of solitary waves for the KdV equation, see Dejak-Sigal [5], Dejak-Jonsson [6], and Holmer [7]. We note that the rigorous derivation of the Schrödinger-KdV system of equations is an open and challenging problem.

The main ingredients of our analysis are generalizations of adiabatic theorems for bound and metastable quantum states to the case at hand, see [10], and also [11-14,16], and a straightforward extension of results on the effective dynamics of solitons for $\mathrm{KdV}$ equation over a slowly varying bottom to the random case, $[7]$, see also $[5,6,8,9]$ for relevant results.

\subsection{The model and statement of the main result}

In what follows, $(\Omega, \mathcal{F}, \mathbb{P})$ denotes a probability triple for which the probability space $\Omega$ has a generic point $\omega$ and is endowed with measure $\mathbb{P}$. For a measurable and integrable function $f$ on $\Omega$, we define its expectation value by $\mathbb{E}(f):=\int f(\omega) \mathbb{P}(d \omega)$.

We consider the system of equations

$$
\begin{aligned}
& i \epsilon \partial_{t} u(x, t)=-\partial_{x}^{2} u(x, t)-\frac{1}{2} g v(x, t) u(x, t) \\
& \partial_{t} v(x, t)=-\partial_{x}\left(\partial_{x}^{2} v(x, t)+3 v^{2}(x, t)-b_{h}(x, t ; \omega) v(x, t)\right)
\end{aligned}
$$

where $\omega \in \Omega$ a point in probability space, $\mathbf{x} \in \mathbb{R}$ denotes a point in the configuration space, $t \in \mathbb{R}$ is time, $g>0$ is a coupling constant, $\epsilon$ and $h$ are small positive paramters, and the slowly varying potential $b_{h}$ is a measurable real function on $\mathbb{R} \times \mathbb{R} \times \Omega \rightarrow \mathbb{R}$ satisfying

$$
b_{h}(\mathbf{x}, t ; \omega) \equiv b(h \mathbf{x}, h t ; \omega) .
$$

We assume that

$$
b \in L^{\infty}\left(\Omega, C_{0}^{\infty}\left(\mathbb{R}^{2}\right)\right) .
$$

Under this assumption, the system of equations (1.1) and (1.2) is almost-surely well-posed in $L^{2}(\mathbb{R}, \mathbb{C}) \times$ $H^{k}(\mathbb{R}, \mathbb{R}), \quad k \geq 1$, for any time interval $[-T, T] \in \mathbb{R}$, see Subsect. 2.1.2 below.

When $b=0$, the KdV equation (1.2) admits orbitally stable solitary wave solutions of the form

$$
v_{\sigma}(x, t)=Q\left(x, a+4 c^{2} t, c\right),
$$

where

$$
\sigma=(a, c), \quad Q(x, a, c)=2 c^{2} \cosh ^{-2}(c(x-a)),
$$

see [17] and references therein. Here, $a$ and $c$ correspond to the centre of mass and the velocity of the internal wave, respectively.

When $g>0$, there exists surface waves that are associated with $v_{\sigma}$. Let

$$
H_{P T}=-\partial_{x}^{2}-g \cosh ^{-2}(x),
$$

the Pöschl-Teller Hamiltonian. It has discrete spectrum

$$
\sigma_{d}\left(H_{P T}\right)=\left\{\lambda_{n}=-\frac{(-2 n-1+\sqrt{1+4 g})^{2}}{4}, \quad n \in \mathbb{N}, \quad n<\frac{-1+\sqrt{1+4 g}}{2}\right\},
$$

see, for example, [18]. We denote by $\chi_{n}$ the eigenfucntion of $H_{P T}$ with eigenvalue $\lambda_{n}$. The surface admits bound states of the form

$$
\chi_{n, \sigma}(x, t)=e^{-i \frac{\lambda_{n}}{\epsilon} \int_{0}^{t} c^{2} d t} e^{i 4 c^{4} \epsilon t} e^{i 2 c^{2} \epsilon\left(x-4 c^{2} t-a\right)} \chi_{n}\left(c\left(x-4 c^{2} t-a\right)\right),
$$

where $n<\frac{-1+\sqrt{1+4 g}}{2}$. These surface waves are bound states of the Hamiltonian $H_{\sigma}=-\partial_{x}^{2}-\frac{1}{2} g v_{\sigma}$. 
The surface waves $\chi_{n, \sigma}$ that are defined above are stable, since they correspond to bound states of a Schrödinger operator. However, in some cases, there exists long-lived metastable surface waves. Suppose that there exists $m \in \mathbb{N}$ and $0<\delta_{g} \ll 1$ such that

$$
m(m+1)-\delta_{g}<g<m(m+1) .
$$

In this case, $\chi_{m}$ is not an eigenstate of $H_{P T}$. However, it is a bound state of $H_{P T}-\delta_{g} \cosh ^{-2} x$. Since the perturbation $\delta_{g} \cosh ^{-2} x$ is dilatation analytic, the results of Theorem 1 in [20] hold, and $\chi_{m}$ can be regarded as an approximate metastable state of $H_{P T}$ with life-time $\tau_{l} \sim \delta_{g}^{-2}$. The approximate metastable surface wave is given by

$$
\chi_{m, \sigma}(x, t)=e^{-i \frac{\lambda m}{\epsilon} \int_{0}^{t} c^{2} d t} e^{i 4 c^{4} \epsilon t} e^{i 2 c^{2} \epsilon\left(x-4 c^{2} t-a\right)} \chi_{m}\left(c\left(x-4 c^{2} t-a\right)\right) .
$$

When $b \neq 0$, the above picture is modified. In the deterministic case, it was shown by Holmer [7] that up to times of order $|\log h| h^{-1}$, the KdV soliton gets modulated due to the slowly varying bottom, see also $[5,6]$. A similar result holds in the random case almost surely, see Theorem 2.1 in Subsect. 2.1.4 for a complete statement.

We introduce the stochastic process $(A(t), C(t))_{t \geq 0}$ corresponding to the rescaled effective dynamics of the centre of mass of the internal wave, which is given by

$$
\left\{\begin{array}{l}
\partial_{t} A(t ; \omega)=4 C^{2}(t ; \omega)-b(A, t ; \omega), \quad A(0)=A_{0}, \\
\partial_{t} C(t ; \omega)=\frac{1}{3} C(t ; \omega) \partial_{A} b(A, t ; \omega), \quad C(0)=C_{0} .
\end{array}\right.
$$

Suppose that $b$ satisfies assumptions (1.3) and (1.4), and that the initial condition of $(1.2)$ is $v(0)=v_{\sigma(0)}$. Then up to times $\tau \sim|\log h| h^{-1}$, the true solution of (1.2) is

$$
v(t)=v_{\sigma(t)}+w(t)
$$

where the fluctuation $w$ is small in a suitable norm to be specified below, and the KdV soliton parameters $\sigma(t)=(a(t), c(t))_{t \geq 0}$ satisfy $\left\|a(t)-h^{-1} A(h t)\right\|_{L_{\omega}^{\infty} L_{[0, \tau]}^{\infty}} \lesssim e^{\alpha h t},\|c(t)-C(h t)\|_{L_{\omega}^{\infty} L_{[0, \tau]}^{\infty}} \lesssim h e^{\alpha h t}$. Here, $\alpha$ is a positive constant that is independent of $h, \epsilon$ and $\omega \in \Omega$.

The bound state for the surface wave is also modulated. Let

$$
H(t)=-\partial_{x}^{2}-\frac{1}{2} g v(t)=H_{\sigma(t)}-\frac{1}{2} g w(t),
$$

where $v_{\sigma}$ and $w$ appear in (1.11). For $h$ small enough, and for each fixed time $t$, the bound states of $H(t)$ can be obtained from those of $H_{\sigma(t)}$ by analytic perturbation theory, since the discrete spectrum of the latter is simple and isolated, see, for example [19]. We denote by $\tilde{\chi}_{n}(t)$ the $n^{\text {th }}$ eigenstate of $H(t)$, $n<\frac{-1+\sqrt{1+4 g}}{2}$, with corresponding eigenvalue $\tilde{\lambda}_{n}(t)$. This eigenstate is an instantaneous bound state. Similarly, one can perturbatively define instantaneous metastable states. Suppose that (1.8) holds. Then $\tilde{\chi}_{m}(t)$, the $m^{t h}$ eigenstate of $H(t)-\frac{1}{2} \delta_{g} v_{\sigma(t)}$, is an approximate metastable state of $H(t)$.

Starting with an initial condition that corresponds to $v_{\sigma_{0}}$ for the internal wave and $\chi_{n, \sigma}\left(\chi_{m, \sigma}\right)$ for the surface wave, one expects the internal wave to be close to $v_{\sigma(t)}$, and the surface wave to be close to the instantaneous bound (metastable) state $\tilde{\chi}_{n}(t)\left(\tilde{\chi}_{m}(t)\right)$. The purpose of this letter is to make this intuition precise, and to give estimates of the deviation from this picture.

We need the notion of stopping time for the dynamics of the internal wave. Suppose $b$ satisfies (1.4), and let $A_{0} \in \mathbb{R}, C_{0}>0$ and $\delta \in(0,1)$. We let $\tau^{*}$ be the stopping time such that

$$
\delta<\|C\|_{L^{\infty}\left(\Omega, L^{\infty}\left(\left[0, \tau^{*}\right]\right)\right)}<\delta^{-1},
$$

where $C$ appears in (1.10). The following is the main result of this work. 
Theorem 1.1. Consider the coupled equations (1.1) and (1.2), such that $b_{h}$ satisfies (1.3) and (1.4). Let $(A(t), C(t))_{t \geq 0}$ be the stochastic process given by (1.10), and define $\tau=\min \left(h^{-1} \tau^{*}, h^{-1} \delta|\log h|\right)$.

(i) Suppose that the initial state is

$$
\left(\begin{array}{l}
u(0) \\
v(0)
\end{array}\right)=\left(\begin{array}{c}
\chi_{n, \sigma_{0}}(0) \\
v_{\sigma_{0}}(0)
\end{array}\right)
$$

where the stable waves $v_{\sigma}$ and $\chi_{n, \sigma}$ are given in (1.5) and (1.7) respectively. Then there exists positive constants $\epsilon_{0}, h_{0}$ and $\alpha$ that depend on the initial condition and $g$, but that are independent of $\omega \in \Omega$, such that, for $t \leq \tau$,

$$
\begin{aligned}
& \mathbb{E}\left(\left\|u-\tilde{\chi}_{n, \sigma(t)}\right\|_{L_{[0, t]}^{\infty} L_{x}^{2}}\right) \lesssim t \epsilon, \\
& \mathbb{E}\left(\left\|v-v_{\sigma(t)}\right\|_{L_{[0, t]}^{\infty} H_{x}^{1}}\right) \lesssim h^{1 / 2} e^{\alpha h t},
\end{aligned}
$$

for $h \in\left(0, h_{0}\right]$ and $\epsilon \in\left(0, \epsilon_{0}\right]$. Here $\sigma(t)=(a(t), c(t))$ satisfy

$$
\begin{aligned}
& \left\|a(t)-h^{-1} A(h t)\right\|_{L_{\omega}^{\infty} L_{[0, t]}^{\infty}} \lesssim e^{\alpha h t}, \\
& \|c(t)-C(h t)\|_{L_{\omega}^{\infty} L_{[0, t]}^{\infty}} \lesssim h e^{\alpha h t} .
\end{aligned}
$$

(ii) Suppose the resonance condition (1.8) holds for some $m \in \mathbb{N}$, and that the initial state is given by

$$
\left(\begin{array}{l}
u(0) \\
v(0)
\end{array}\right)=\left(\begin{array}{c}
\chi_{m, \sigma_{0}}(0) \\
v_{\sigma_{0}}(0)
\end{array}\right)
$$

where the approximate metastable state $\chi_{m, \sigma}$ is given in (1.9). Then there exists positive constants $\epsilon_{0}$, $h_{0}, \delta_{g_{0}}$ and $\alpha$ that depend on the initial condition and $g$, but that are independent of $\omega \in \Omega$, such that, for $t \leq \tau$,

$$
\begin{aligned}
& \mathbb{E}\left(\left\|u-\tilde{\chi}_{m, \sigma(t)}\right\|_{L_{[0, t]}^{\infty} L_{x}^{2}}\right) \lesssim\left(\delta_{g} \epsilon^{-1}+\epsilon^{1 / 2}+f(\epsilon)\right) t, \\
& \mathbb{E}\left(\left\|v-v_{\sigma(t)}\right\|_{L_{[0, t]}^{\infty} H_{x}^{1}}\right) \lesssim h^{1 / 2} e^{\alpha h t},
\end{aligned}
$$

for $h \in\left(0, h_{0}\right], \epsilon \in\left(0, \epsilon_{0}\right]$ and $\delta_{g} \in\left(0, \delta_{g_{0}}\right]$. Here, $f(x) \rightarrow 0$ and $x \rightarrow 0$, and $\sigma(t)=(a(t), c(t))$ satisfy

$$
\begin{aligned}
& \left\|a(t)-h^{-1} A(h t)\right\|_{L_{\omega}^{\infty} L_{[0, t]}^{\infty}} \lesssim e^{\alpha h t}, \\
& \|c(t)-C(h t)\|_{L_{\omega}^{\infty} L_{[0, t]}^{\infty}} \lesssim h e^{\alpha h t} .
\end{aligned}
$$

Note that in case (i), when $\epsilon \sim \frac{h^{3 / 2-\delta \alpha}}{\delta|\log h|}$, the deviation of the surface wave and the internal wave from the coupled bound state is of order $O\left(h^{1 / 2-\delta \alpha}\right)$.

\subsection{Organization}

The organization of this letter is as follows. In Sect. 2, we briefly recall basic properties of (1.1) and (1.2). We also discuss the effective dynamics of the internal wave. In Sect. 3, we state and prove the adiabatic theorems corresponding to the evolution of the surface wave. Finally, in Sect. 4, we prove Theorem 1.1.

\subsection{Notation}

- In the following, $L^{p}(I)$ denotes the (real or complex) Lebesgue space, $1 \leq p \leq \infty$, with norm

$$
\begin{aligned}
& \|f\|_{L^{p}}=\left(\int_{I} d x|f(x)|^{p}\right)^{\frac{1}{p}}, \quad f \in L^{p}(I), p<\infty, \\
& \|f\|_{L^{\infty}}=\operatorname{ess}-\sup (|f|), \quad f \in L^{\infty}(I) .
\end{aligned}
$$


The ordering of norms is standard. For example,

$$
\|f\|_{L_{\omega}^{\infty} L_{[0, T]}^{\infty}}:=\|\| f\left\|_{L^{\infty}([0, T])}\right\|_{L^{\infty}(\Omega)} .
$$

We denote by $\langle\cdot, \cdot\rangle$ the scalar product in $L^{2}(\mathbb{R})$,

$$
\langle u, v\rangle=\left\{\begin{array}{l}
\operatorname{Re} \int_{\mathbb{R}} u \bar{v}, \quad u, v \in L^{2}(\mathbb{R}, \mathbb{C}) \\
\int_{\mathbb{R}} u v, \quad u, v \in L^{2}(\mathbb{R}, \mathbb{R})
\end{array}\right.
$$

- We denote by

$$
\begin{aligned}
& H^{s}(\mathbb{R}):=\left\{u \in \mathcal{S}^{\prime}(\mathbb{R}): \mathcal{F}^{-1}\left(1+|k|^{2}\right)^{\frac{s}{2}} \mathcal{F} u \in L^{2}(\mathbb{R})\right\}, \\
& s \in \mathbb{R},
\end{aligned}
$$

where $\mathcal{F}$ stands for the Fourier transform. The space $H^{s}$ is equipped with the norm

$$
\|u\|_{H^{s}}=\left\|\mathcal{F}^{-1}\left(1+|k|^{2}\right)^{\frac{s}{2}} \mathcal{F} u\right\|_{L^{2}}, \quad u \in H^{s}(\mathbb{R}),
$$

which makes it a (real or complex) Banach space.

- For $1 \leq p \leq \infty$ and $s \in \mathbb{N}$, the (real or complex) Sobolev space is given by

$$
W^{s, p}(\mathbb{R}):=\left\{u \in \mathcal{S}^{\prime}(\mathbb{R}): \partial_{x}^{\bar{\alpha}} u \in L^{p}(\mathbb{R}),|\bar{\alpha}| \leq s\right\},
$$

where $\mathcal{S}^{\prime}(\mathbb{R})$ is the space of tempered distributions. We equip $W^{s, p}$ with the norm

$$
\|u\|_{W^{s, p}}=\sum_{\alpha,|\alpha| \leq s}\left\|\partial_{x}^{\bar{\alpha}} u\right\|_{L^{p}}
$$

which makes it a Banach space.

\section{Preliminaries}

\subsection{Modified KdV equation}

We now recall some of the properties of the modified KdV equation (1.2); see, for example, [5], [6] and [7]. In what follows, we denote $\bar{\Omega} \subset \Omega$, with $\mathbb{P}(\bar{\Omega})=1$, the set over which the realizations of $b$ are uniformly bounded in $\omega$ and are in $C_{0}^{\infty}\left(\mathbb{R}^{2}\right)$.

\subsubsection{Hamiltonian and symplectic structure}

The Hamiltonian functional associated with (1.2) is

$$
H_{b}(v)=\frac{1}{2} \int_{\mathbb{R}}\left[\left(\partial_{x} v\right)^{2}-2 v^{3}+b_{h} v^{2}\right] d x .
$$

Since $b_{h}$ is time-dependent, $H_{b}$ is not conserved. In particular,

$$
\partial_{t} H_{b}(v)=\frac{1}{2} \int_{\mathbb{R}} \partial_{t} b_{h} v^{2}, \quad \mathbb{P}-\text { a.s.. }
$$

Furthermore, since translational symmetry is broken when $b \neq 0$, the momentum $m(v)=\frac{1}{2}\|v\|_{L^{2}}^{2}$ is no more conserved, and

$$
\partial_{t} m(v)=\frac{1}{2} \int_{\mathbb{R}} \partial_{x} b_{h} v^{2}, \quad \mathbb{P}-\text { a.s.. }
$$


Let $J=\partial_{x}$, then

$$
J^{-1} v(x)=\frac{1}{2}\left(\int_{-\infty}^{x} v\left(x^{\prime}\right) d x^{\prime}-\int_{x}^{+\infty} v\left(x^{\prime}\right) d x^{\prime}\right)
$$

defines a symplectic form on $H^{1}(\mathbb{R}, \mathbb{R})$ that is given by

$$
\omega(v, w)=\left\langle v, J^{-1} w\right\rangle
$$

The modified KdV equation can be regarded as the Hamiltonian flow generated by $H_{b}$ :

$$
\partial_{t} v=J H_{b}^{\prime}(v)
$$

where the prime stands for the Fréchet derivative.

\subsubsection{Well-posedness}

Under assumptions (1.3) and (1.4), the modified KdV equation (1.2) is $\mathbb{P}$-a.s. globally well-posed in $H^{1}(\mathbb{R}, \mathbb{R})$. This follows directly from the well-posedness result in the deterministic case in the presence of a bottom, see $[5,7]$. For fixed $\omega \in \bar{\Omega}$, local well-posedness follows from smoothing estimates of Kenig-Ponce-Vega [21]. When $b \neq 0$, the momentum $m(v)$ and the Hamiltonian $H_{b}(v)$ are not conserved. However, it follows from (2.2) and (2.3) that they are almost surely approximately conserved. This implies that (1.2) is almost surely globally well-posed in $H^{1}(\mathbb{R}, \mathbb{R})$. Since the term with the highest derivative is linear, and $b$ is almost surely in $C_{0}^{\infty}\left(\mathbb{R}^{2}\right)$, the analysis can be extended to $H^{k}(\mathbb{R}, \mathbb{R}), k \geq 1$, for any time interval $[-T, T] \in \mathbb{R}$.

\subsubsection{Internal wave manifold}

When $b=0,(1.2)$ admits solitary wave solutions of the form

$$
v_{\sigma}(x, t)=Q\left(x, a+4 c^{2} t, c\right)=2 c^{2} \cosh ^{-2}\left(x-4 c^{2} t-a\right), \quad a \in \mathbb{R}, \quad c>0 .
$$

Let $\mathcal{M} \subset H^{1}$ denote the two-dimensional soliton manifold

$$
\mathcal{M}=\left\{v_{\sigma} \mid \quad \sigma=(a, c) \in \mathbb{R} \times \mathbb{R}^{+}\right\} .
$$

The symplectic form $\omega$ when restricted to the soliton manifold is

$$
\left.\omega\right|_{\mathcal{M}}=8 c^{2} d a \wedge d c
$$

and the Hamiltonian restricted to $\mathcal{M}$ is

$$
\left.H_{b}\right|_{\mathcal{M}}=-\frac{32}{5} c^{5}+\frac{1}{2} \int_{\mathbb{R}} b_{h} v_{\sigma}^{2} d x .
$$

In the deterministic case, it was shown in [7] that the effective dynamics of the centre of mass of the soliton is approximately given by the Hamiltonian flow generated by $\left.H_{b}\right|_{\mathcal{M}}$. A similar result holds in the case when $b$ is random, yet slowly varying.

\subsubsection{Long-time dynamics of internal wave}

Since $b \in C_{0}^{\infty}\left(\mathbb{R}^{2}\right)$ when $\omega \in \bar{\Omega}$, the result of Theorem 2 in [7] holds almost surely.

Theorem 2.1 (Effective dynamics of internal wave). Consider equation (1.2), such that $b_{h}$ satisfies (1.3) and (1.4). Let $(A(t), C(t))_{t \geq 0}$ be the stochastic process given by (1.10), and define $\tau=$ $\min \left(h^{-1} \tau^{*}, h^{-1} \delta|\log h|\right)$. Suppose that

$$
v(0)=v_{\sigma}(0),
$$


where the stable wave $v_{\sigma}$ is given in (1.5). Then there exists positive constants $h_{0}$ and $\alpha$ such that, for $t \leq \tau$,

$$
\begin{aligned}
& \mathbb{E}\left(\left\|v-v_{\sigma(t)}\right\|_{L_{[0, t]}^{\infty} H_{x}^{1}}\right) \lesssim h^{1 / 2} e^{\alpha h t} \\
& \left\langle w, v_{\sigma(t)}\right\rangle=\left\langle w,(x-a) v_{\sigma}\right\rangle=0 \quad \mathbb{P}-\text { a.s. }
\end{aligned}
$$

for $h \in\left(0, h_{0}\right]$. Here, $\sigma(t)=(a(t), c(t))$ satisfy

$$
\begin{aligned}
& \left\|a(t)-h^{-1} A(h t)\right\|_{L_{\omega}^{\infty} L_{[0, t]}^{\infty}} \lesssim e^{\alpha h t}, \\
& \|c(t)-C(h t)\|_{L_{\omega}^{\infty} L_{[0, t]}^{\infty}} \lesssim h e^{\alpha h t} .
\end{aligned}
$$

The proof in [7] relies on the geometric approach to study effective dynamics of solitons as developed in [22] for the case of the nonlinear Schrödinger equation, see also [5,6] for earlier results. When $v$ is close enough (in $H^{1}$ norm) to the soliton manifold $\mathcal{M}$, the implicit function theorem implies that there exists a unique orthogonal decomposition

$$
v=v_{\sigma}+w,
$$

where the fluctuation $w$ satisfies the orthogonality conditions

$$
\left\langle w, v_{\sigma}\right\rangle=\left\langle w,(x-a) v_{\sigma}\right\rangle=0 .
$$

The equation of motion for $w$ is

$$
\partial_{t} w=-\partial_{x}\left(\partial_{x}^{2} w+6 v_{\sigma} w-b w+3 w^{2}\right)+F_{0},
$$

where

$$
F_{0}=-\left(\partial_{t} a-4 c^{2}\right) \partial_{a} v_{\sigma}-\left(\partial_{t} c\right) v_{\sigma}+\partial_{x}\left(b v_{\sigma}\right)
$$

Control of the fluctuation is achieved using the Martel-Merle local virial identity, [23], and energy estimates. Assumption (1.4), almost sure well-posedness in $H^{k}, k \geq 1,(2.4)$ and the orthogonality conditions imply that

$$
a, c \in L^{\infty}\left(\Omega, W^{2, \infty}(\mathbb{R})\right)
$$

\subsection{The surface equation}

In this subsection, we review some of the properties of (1.1). Suppose that the hypotheses of Theorem 2.1 hold, and let

$$
H_{\sigma(t)}=-\partial_{x}^{2}-\frac{1}{2} g v_{\sigma(t)}, \quad t \leq \tau .
$$

Since $v_{\sigma}$ is almost surely bounded and smooth, $H_{\sigma(t)}$ is $\mathbb{P}$-a.s. ess. self-adjoint with fixed common core. Let

$$
H(t)=-\partial_{x}^{2}-\frac{1}{2} g v(t)=H_{\sigma(t)}-\frac{1}{2} g w(t) .
$$

It follows from the well-posedness of $(1.2)$ with initial data $v_{\sigma_{0}} \in H^{k}(\mathbb{R}), k \geq 1$, that $\frac{1}{2} g w(t)$ is $\mathbb{P}$-a.s. bounded perturbation, and hence, $H(t)$ is $\mathbb{P}$-a.s. self-adjoint on a fixed dense domain $\mathcal{D}$. Furthermore, the discrete spectrum of $H(t)$ is obtained from that of $H_{\sigma(t)}$ using standard perturbation theory. We denote by $P_{n}(t)=\left|\tilde{\chi}_{n}(t)\right\rangle\left\langle\tilde{\chi}_{n}(t)\right|$ the eigenprojection corresponding to the isolated simple eigenvalue $\tilde{\lambda}_{n}(t)$ of $H(t), n<\frac{-1+\sqrt{1+4 g}}{2}$. Furthermore, we let

$$
R(t, z)=(z-H(t))^{-1},
$$


the resolvent of $H(t)$. Well-posedness of (1.2) and (2.5) imply that $R(t, i)$ is $\mathbb{P}$-a.s. bounded and differentiable as a bounded operator, and that $H(t) \partial_{t} R(t, i)$ is $\mathbb{P}$-a.s. bounded. Note that one can define $P_{n}$ using the resolvent and contour integration,

$$
P_{n}(t)=\frac{1}{2 \pi i} \int_{\gamma} R(t, z) d z
$$

where $\gamma$ is a contour that encloses $\tilde{\lambda}_{n}(t)$. It follows that $P_{n}(t)$ is $\mathbb{P}$-a.s. twice differentiable as a bounded operator. In what follows, we denote by $U$ the a.s. unitary operator that is given by

$$
i \epsilon \partial_{t} U\left(t, t^{\prime}\right)=H(t) U\left(t, t^{\prime}\right), \quad U(t, t)=1 .
$$

\section{Adiabatic evolution of surface waves}

\subsection{Adiabatic evolution of bound surface waves}

In this subsection, we study the dynamics of the surface wave when the initial condition is close to a stable bound state. Following the analysis of Kato [11], we introduce the generator of the adiabatic evolution $U_{a}\left(t, t^{\prime}\right)$ that satisfies the intertwining property

$$
U_{a}(t, 0) P_{n}(0)=P_{n}(t) U_{a}(t, 0) \mathbb{P}-\text { a.s. }
$$

Let

$$
H_{a}(t)=H(t)+i \epsilon\left[\dot{P}_{n}(t), P_{n}(t)\right]
$$

where $\dot{f}=\partial_{t} f$. This operator is $\mathbb{P}$-a.s. self-adjoint and differentiable in $t$. Furthermore, using the fact that

$$
P_{n}(t) \dot{P}_{n}(t) P_{n}(t)=0, \quad \mathbb{P}-\text { a.s. },
$$

one can directly verify that $H_{a}(t)$ generates $U_{a}(t)$,

$$
i \epsilon \partial_{t} U_{a}\left(t, t^{\prime}\right)=H_{a}(t) U_{a}\left(t, t^{\prime}\right), \quad U_{a}(t, t)=1 .
$$

$U_{a}$ is $\mathbb{P}$-a.s. unitary.

Theorem 3.1. Suppose that the hypotheses of Theorem 2.1 hold. Then

$$
\left\|U(t, 0)-U_{a}(t, 0)\right\| \lesssim \epsilon t, \quad \mathbb{P}-\text { a.s. }
$$

uniformly in $h \in\left(0, h_{0}\right]$.

Proof. We want to compare $U_{a}(t, 0)$ to $U(t, 0)$. Let $\omega \in \bar{\Omega}$ and

$$
\delta(t)=U_{a}^{*}(t, 0) U(t, 0) .
$$

Then

$$
\begin{aligned}
\partial_{t} \delta(t) & =\frac{i}{\epsilon} U_{a}^{*}(t, 0)\left(H_{a}(t)-H(t)\right) U(t, 0) \\
& =-U_{a}^{*}(t, 0)\left[\dot{P}_{n}(t), P_{n}(t)\right] U(t, 0) .
\end{aligned}
$$

Let

$$
X(t)=\frac{1}{2 \pi i} \int_{\gamma} R(t, z) \dot{P}_{n}(t) R(t, z)
$$


where $\gamma$ is a contour in the complex plane that encloses $\tilde{\lambda}_{n}(t), t \in[0, \tau]$. Note that $X$ is $\mathbb{P}$-a.s. differentiable in $t$ as a bounded operator. It also satisfies the commutator equation

$$
[H(t), X(t)]=\left[\dot{P}_{n}(t), P_{n}(t)\right], \quad \mathbb{P}-\text { a.s.. }
$$

It follows that

$$
\begin{aligned}
\partial_{t} \delta(t) & =U_{a}^{*}(t, 0)[X(t), H(t)] U(t, 0) \\
& =U_{a}^{*}(t, 0)\left(X(t) H(t)-H_{a}(t) X(t)\right) U(t, 0)+O(\epsilon) \\
& =-i \epsilon\left[\left(\partial_{t} U_{a}^{*}(t, 0)\right) X(t) U(t, 0)-U_{a}^{*}(t, 0) X(t)\left(\partial_{t} U(t, 0)\right)\right]+O(\epsilon) \\
& =-i \epsilon\left[\partial_{t}\left(U_{a}^{*}(t, 0) X(t) U(t, 0)\right)-U_{a}^{*}(t, 0)\left(\partial_{t} X(t)\right) U(t, 0)\right]+O(\epsilon) .
\end{aligned}
$$

Since $X$ and $\partial_{t} X$ are $\mathbb{P}$-a.s. bounded,

$$
\left\|\partial_{t} \delta(t)\right\| \lesssim \epsilon, \quad \mathbb{P}-\text { a.s. }
$$

uniformly in $t \in[0, \tau]$. This yields

$$
\|\delta(t)-1\| \lesssim \epsilon t, \quad \mathbb{P}-\text { a.s.. }
$$

\subsection{Adiabatic evolution of metastable surface waves}

We now assume that the resonance condition (1.8) holds for some $m \in \mathbb{N}$, and that the initial condition of (1.1) is the approximate metastable state $u_{0}=\chi_{m, \sigma_{0}}(0)$. We have a weaker form of the adiabatic theorem compared to that of the bound state.

Let

$$
H_{0}(t)=H(t)-\frac{1}{2} \delta_{g} v_{\sigma(t)}
$$

Then, for each fixed $t \in[0, \tau]$ and $\omega \in \bar{\Omega}, \tilde{\lambda}_{m}(t)$ is a simple isolated eigenvalue of $H_{0}(t)$. Let $P_{m}(t)=\left|\tilde{\chi}_{m}(t)\right\rangle\left\langle\tilde{\chi}_{m}(t)\right|$, the eigenprojection corresponding to $\tilde{\lambda}_{m}(t)$. We introduce the generator of the approximate adiabatic evolution

$$
H_{a}^{0}(t):=H(t)+i \epsilon\left[\dot{P}_{m}(t), P_{m}(t)\right]
$$

which is $\mathbb{P}$-a.s. self-adjoint with the same common dense domain $\mathcal{D}$ of $H(t)$. The propagator corresponding to the approximate adiabatic evolution is given by

$$
i \epsilon \partial_{t} U_{a}^{0}\left(t, t^{\prime}\right)=H_{a}^{0}(t) U_{a}^{0}\left(t, t^{\prime}\right), U_{a}^{0}(t, t)=1 .
$$

$U_{a}^{0}$ is a $\mathbb{P}$-a.s. unitary operator.

Theorem 3.2. Suppose that the hypotheses of Theorem 2.1 and (1.8) hold. Then, for $t \in[0, \tau]$,

$$
U_{a}^{0}(t, 0) P_{m}(0) U_{a}^{0}(0, t)=P_{m}(t)+O\left(\delta_{g} \epsilon^{-1} t\right), \quad \mathbb{P}-\text { a.s. },
$$

and

$$
\left\|U(t, 0)-U_{a}^{0}(t, 0)\right\| \lesssim\left[\epsilon^{1 / 2}+\delta_{g} \epsilon^{-1 / 4}+l\left(\epsilon^{1 / 4}\right)\right] t, \quad \mathbb{P}-\text { a.s. },
$$

uniformly in $h \in\left(0, h_{0}\right]$, where $l(x)$ is a positive function of $x \in \mathbf{R}$ such that $\lim _{x \rightarrow 0} l(x)=0$. 
Proof. Let $\omega \in \bar{\Omega}$ and define

$$
h\left(t, t^{\prime}\right)=U_{a}^{0}\left(t, t^{\prime}\right) P_{m}\left(t^{\prime}\right) U_{a}^{0}\left(t^{\prime}, 0\right)
$$

Then

$$
\begin{aligned}
\partial_{s^{\prime}} h\left(t, t^{\prime}\right) & =i \epsilon^{-1} U_{a}^{0}\left(t, t^{\prime}\right)\left\{H_{a}^{0}\left(t^{\prime}\right) P_{m}\left(t^{\prime}\right)-P_{m}\left(t^{\prime}\right) H_{a}^{0}\left(t^{\prime}\right)\right\} U_{a}^{0}\left(t^{\prime}, 0\right) \\
& =i \epsilon^{-1} U_{a}^{0}\left(t, t^{\prime}\right)\left\{\lambda_{m}\left(t^{\prime}\right) P_{m}\left(t^{\prime}\right)+i \epsilon \dot{P}_{m}\left(t^{\prime}\right) P_{m}\left(t^{\prime}\right)-\lambda_{m}\left(t^{\prime}\right) P_{m}\left(t^{\prime}\right)\right. \\
& \left.+i \epsilon P_{m}\left(t^{\prime}\right) \dot{P}_{m}\left(t^{\prime}\right)+O\left(\delta_{g}\right)\right\} U_{a}^{0}\left(t^{\prime}, 0\right) \\
& =O\left(\delta_{g} \epsilon^{-1}\right), \quad \omega \in \bar{\Omega},
\end{aligned}
$$

where we have used the definition of the generator of the adiabatic evolution and the property that

$$
\dot{P}_{m}(t) P_{m}(t)+P_{m}(t) \dot{P}_{m}(t)=0, \quad \mathbb{P}-\text { a.s.. }
$$

It follows that $h(t, 0)=h(t, t)+O\left(\delta_{g} \epsilon^{-1} t\right), \quad \mathbb{P}-$ a.s., and hence claim (3.4).

For $\psi \in \mathcal{D}$ and $\omega \in \bar{\Omega}$, we have

$$
\begin{aligned}
\left(U(t, 0)-U_{a}^{0}(t, 0)\right) \psi & =-\int_{0}^{t} d t^{\prime} \partial_{t^{\prime}}\left(U\left(t, t^{\prime}\right) U_{a}^{0}\left(t^{\prime}, 0\right)\right) \psi \\
& =-i \epsilon^{-1} \int_{0}^{t} d t^{\prime} U\left(t, t^{\prime}\right)\left[H\left(t^{\prime}\right)-H_{a}^{0}\left(t^{\prime}\right)\right] U_{a}^{0}\left(t^{\prime}, 0\right) \psi \\
& =-\int_{0}^{t} d t^{\prime} U\left(t, t^{\prime}\right)\left[\dot{P}_{m}\left(t^{\prime}\right), P_{m}\left(t^{\prime}\right)\right] U_{a}^{0}\left(t^{\prime}, 0\right) \psi
\end{aligned}
$$

Since the domain of definition $\mathcal{D}$ is dense, it follows that

$$
\left\|U(t, 0)-U_{a}^{0}(t, 0)\right\|=\left\|\int_{0}^{t} d t^{\prime} U\left(t, t^{\prime}\right)\left[\dot{P}_{m}\left(t^{\prime}\right), P_{m}\left(t^{\prime}\right)\right] U_{a}^{0}\left(t^{\prime}, 0\right)\right\|, \quad \mathbb{P}-\text { a.s.. }
$$

For $\omega \in \bar{\Omega}$, let

$$
X_{\eta}(t):=R\left(\lambda_{m}(t)+i \eta, t\right) \dot{P}_{m}(t) P_{m}(t)+P_{m}(t) \dot{P}_{m}(t) R\left(\lambda_{m}(t)-i \eta, t\right) .
$$

Note that

$$
\begin{aligned}
{\left[H(t), X_{\eta}(t)\right] } & =\left[H(t)-\lambda_{m}(t)-i \eta, R\left(\lambda_{m}(t)+i \eta, t\right) \dot{P}_{m}(t) P_{m}(t)\right] \\
& +\left[H(t)-\lambda_{m}(t)+i \eta, P_{m}(t) \dot{P}_{m}(t) R\left(\lambda_{m}(t)-i \eta, t\right)\right] \\
& =\left[\dot{P}_{m}(t), P_{m}(t)\right]+i \eta X_{\eta}(t)+O\left(\delta_{g} / \eta\right) .
\end{aligned}
$$

Furthermore,

$$
\begin{aligned}
\partial_{t^{\prime}}\left(U\left(t, t^{\prime}\right) X_{\eta}\left(t^{\prime}\right) U_{a}^{0}\left(t^{\prime}, 0\right)\right) & =i \epsilon^{-1} U\left(t, t^{\prime}\right)\left[H\left(t^{\prime}\right), X_{\eta}\left(t^{\prime}\right)\right] U_{a}^{0}\left(t^{\prime}, 0\right) \\
& +U\left(t, t^{\prime}\right) X_{\eta}\left(t^{\prime}\right)\left[\dot{P}_{m}\left(t^{\prime}\right), P_{m}\left(t^{\prime}\right)\right] U_{a}^{0}\left(t^{\prime}, 0\right) \\
& +U\left(t, t^{\prime}\right) \dot{X}_{\eta}\left(t^{\prime}\right) U_{a}^{0}\left(t^{\prime}, 0\right) .
\end{aligned}
$$

This yields

$$
\begin{aligned}
& \left\|\int_{0}^{t} d t^{\prime} U\left(t, t^{\prime}\right)\left[\dot{P}_{m}\left(t^{\prime}\right), P_{m}\left(t^{\prime}\right)\right] U_{a}^{0}\left(t^{\prime}, 0\right)\right\| \\
& \leq \sup _{t}\left\{\epsilon\left[\left\|X_{\eta}(t)\right\|\left(1+2\left\|\dot{P}_{m}(t) P_{m}(t)\right\|\right)+\left\|\dot{X}_{\eta}(t)\right\|\right]+\eta\left\|X_{\eta}(t)\right\|\right\}+C \delta_{g} / \eta
\end{aligned}
$$


for $\omega \in \bar{\Omega}$, where $C$ is a finite constant independent of $h, \epsilon$ and $\delta_{g}$. We claim that the following estimates are $\mathbb{P}$-a.s. true for small enough $\eta$ and $\delta_{g}$,

$$
\begin{aligned}
& \text { (i) }\left\|X_{\eta}(t)\right\|<C / \eta \mathbb{P}-\text { a.s., } \\
& \text { (ii) }\left\|\dot{X}_{\eta}(t)\right\|<C / \eta^{2} \mathbb{P}-\text { a.s., } \\
& \text { (iii) } \eta\left\|X_{\eta}(t)\right\|<B(\eta)+C \delta_{g} / \eta, \quad \mathbb{P} \text { - a.s., }
\end{aligned}
$$

where $\lim _{\eta \rightarrow 0} B(\eta)=0$, and $C$ is a finite constant independent of $h, \epsilon$ and $\delta_{g}$.

Estimates $(i)$ and (ii) follow from our knowledge of the spectrum of $H(t)$ when $\omega \in \bar{\Omega}$ and the resolvent identity. We now compare the L.H.S. of (3.9) to the "unperturbed case". Let $R_{0}(t, z)=\left(z-H_{0}(t)\right)^{-1}$, where the "unperturbed" Hamiltonian $H_{0}=H-\frac{1}{2} \delta_{g} v_{\sigma}$. Let

$$
\tilde{X}_{\eta}(t):=R_{0}\left(\lambda_{m}(t)+i \eta, t\right) \dot{P}_{m}(t) P_{m}(t)+P_{m}(t) \dot{P}_{m}(t) R_{0}\left(\lambda_{m}(t)-i \eta, t\right) .
$$

By the second resolvent identity,

$$
\left\|X_{\eta}(t)\right\| \leq\left\|\tilde{X}_{\eta}(t)\right\|+C \delta_{g} / \eta^{2}, \mathbb{P}-\text { a.s. }
$$

uniformly in $h, \epsilon$ and $\delta_{g}$. We claim that

$$
\lim _{\eta \rightarrow 0} \eta^{2}\left\|\tilde{X}_{\eta}(t)\right\|^{2}=0
$$

Consider $\phi \in \mathcal{D}$, then $\psi(t)=\dot{P}_{m}(t) P_{m}(t) \phi \in \operatorname{Ran}\left(1-P_{m}(t)\right)$. Using the spectral theorem for $H_{0}(t)$, we have the following for every $\omega \in \bar{\Omega}$,

$$
\begin{aligned}
& \lim _{\eta \rightarrow 0} \eta^{2}\left\|R_{0}\left(\lambda_{m}(t)+i \eta, t\right) \dot{P}_{m}(t) P_{m}(t) \phi\right\|^{2} \\
& =\lim _{\eta \rightarrow 0} \eta^{2}\left\langle\psi(t), R_{0}\left(\lambda_{m}(t)-i \eta, t\right) R_{0}\left(\lambda_{m}(t)+i \eta, t\right) \psi(t)\right\rangle \\
& =\lim _{\eta \rightarrow 0} \eta^{2} \int d \mu_{\psi(t)}(\lambda) \frac{1}{\left(\lambda-\lambda_{m}(t)\right)^{2}+\eta^{2}} \\
& =\mu\left(\psi(t) \in \operatorname{Ran}\left(P_{m}(t)\right)\right)=0,
\end{aligned}
$$

and hence claim (3.11). Therefore,

$$
\left\|U(t, 0)-U_{a}^{0}(t, 0)\right\| \leq C_{1} \frac{\epsilon}{\eta^{2}}+C_{2} \frac{\delta_{g}}{\eta}+l(\eta),
$$

where $C_{1,2}$ are finite constants, and $\lim _{\eta \rightarrow 0} l(\eta)=0$. Choosing $\eta=\epsilon^{1 / 4}$ yields $(3.5)$.

\section{Proof of main result}

Proof. of Theorem 1.1. The main theorem follows directly from Theorems 2.1, 3.1 and 3.2.

(i) It follows from Theorem 3.1 that

$$
\begin{aligned}
\left\|u(t)-\tilde{\chi}_{n}(t)\right\| & =\left\|\left(U(t, 0)-U_{a}(t, 0)\right) \chi_{n, \sigma_{0}}(0)\right\| \\
& \lesssim \epsilon t, \quad \mathbb{P}-\text { a.s. },
\end{aligned}
$$

uniformly in $h$. Together with Theorem 2.1, this implies (i). 
(ii) It follows from Theorem 3.2 that

$$
\begin{aligned}
\left\|u(t)-\tilde{\chi_{m}}(t)\right\| & \lesssim\left\|\left(U(t, 0)-U_{a}^{0}(t, 0)\right) \chi_{m, \sigma_{0}}(0)\right\|+O\left(\delta_{g} \epsilon^{-1} t\right) \\
& \lesssim\left(\epsilon^{1 / 2}+\delta_{g} \epsilon^{-1}+f(\epsilon)\right) t, \quad \mathbb{P}-\text { a.s. },
\end{aligned}
$$

uniformly in $h$. Together with Theorem 2.1, this implies (ii).

Acknowledgements. The financial support of a Discovery grant from the Natural Sciences and Engineering Research Council of Canada is gratefully acknowledged.

\section{References}

[1] W. Craig, P. Guyenne, C. Sulem. Coupling between internal and surface waves, Natural Hazards, Special Issue on "Internal waves in the oceans and estuaries: modeling and observations", (2010), doi:10.1007/s11069-010-9535-4, 26pp.

[2] W. Craig, P. Guyenne, C. Sulem. A Hamiltonian approach to nonlinear modulation analysis. Wave Motion 47 (2010), 552-563.

[3] E. van Groesen, S. R. Pudjaprasetya. Uni-directional waves over slowly varying bottom. I. Derivation of a KdV-type of equation. Wave Motion 18 (1993), 345-370.

[4] S. B. Yoon, Philip L.-F. Liu. A note on Hamiltonian for long water waves in varying depth. Wave Motion 20 (1994), 359-370.

[5] S.I Dejak, I.M. Segal. Long time dynamics of KdV solitary waves over a variable bottom. Comm. Pure Appl. Math. 59 (2006), 869-905.

[6] S.I. Dejak, B.L.G Jonsson. Long time dynamics of variable coefficient mKdV solitary waves. J. Math. Phys. 47 (2006), 072703, 16pp.

[7] J. Holmer. Dynamics of KdV solitons in the presence of a slowly varying potential. IMRN (2011), doi:10.1093/imrn/rnq284, 31pp.

[8] J. Holmer, G. Perelman, M. Zworski. Effective dynamics of double solitons for perturbed mKdV. Commun. Math. Phys. 305 (2011), 363-425.

[9] C. Munoz. On the soliton dynamics under a slowly varying medium for generalized KdV equations. arxiv.org arXiv:0912.4725 [math.AP] (2009). To appear in Analysis and PDE.

[10] W. Abou Salem, J. Fröhlich. Adiabatic theorems for quantum resonances. Commun. Math. Phys. 273 (2007), $651-675$.

[11] T. Kato. On the adiabatic theorem of quantum mechanics. Phys. Soc. Jap. 5 (1958), 435-439.

[12] J.E. Avron, A. Elgart. Adiabatic theorem without a gap condition. Commun. Math. Phys. 203 (1999), $445-463$.

[13] S. Teufel. A note on the adiabatic theorem without a gap condition. Lett. Math. Phys. 58 (2002), 261-266.

[14] A. Joye. General adiabatic evolution with a gap condition. Commun. Math. Phys. 275 (2007), 139-162.

[15] V. S. Buslaev, C. Sulem. Linear adiabatic dynamics generated by operators with continuous spectrum. Asymptotic Anal. 58 (2008), 17-45.

[16] A. Elgart, G. A. Hagedorn. An adiabatic theorem for resonances. Comm. Pure Appl. Math. 64 (2011), $1029-1058$.

[17] J.L. Bona, P.E. Souganidis, W.A. Strauss. Stability and instability of solitary waves of Korteweg de Vries type. Proc. Roy. Soc. London Ser. A 411 (1987), 395-412.

[18] L. Guillopé, M. Zworski. Upper bounds on the number of resonances on noncompact Riemann e surfaces. J. Func. Anal. 129 (1995), 364-389.

[19] T. Kato. Perturbation Theory for Linear Operators. Springer-Verlag New York, 1991.

[20] W. Hunziker. Resonances, metastable states and exponential decay laws in perturbation theory. Commun. Math. Phys. 132 (1990), 177.

[21] C.E. Kenig, G. Ponce, L. Vega. Well-posedness and scattering results for the generalized Korteweg-de Vries equation via the contraction principle. Comm. Pure Appl. Math. 46 (1993), 527-620.

[22] J. Holmer, M. Zworski. Soliton interaction with slowly varying potentials. IMRN (2008), doi: 10.1093/imrn/rnn026, 36 pp.

[23] Y. Martel, F. Merle. Asymptotic stability of solitons for subcritical gKdV equations revisited. Nonlinearity 18 (2005), 55-80. 